Orthopäde 2009 · 38:785-785

DOI 10.1007/s00132-009-1464-0

Online publiziert: 3. September 2009

(c) Springer Medizin Verlag 2009

\author{
B.-D. Katthagen \\ Orthopädische Klinik, Klinikum Dortmund
}

\title{
Begleiterkrankungen und Komplikationen in der orthopädischen Chirurgie
}

Asepsis, moderne Anästhesieverfahren und möglichst atraumatische Chirurgie mit leistungsstarken, gut verträglichen Implantaten und Materialien durch erfahrene, geschulte und geübte Chirurgen ermöglichen heute regelmäßig früher unglaubliche Erfolge in der Rekonstruktionschirurgie des Bewegungsapparates vom Säugling bis ins hohe Alter. Wenn nur die Komplikationen nicht wären!

Wir sind es gewöhnt, die orthopädische Chirurgie und Unfallchirurgie systematisch nach der Krankheitslehre und den Operationsverfahren zu analysieren und betrachten. Den lästigen Komplikationen wird dabei meist nur ein Nebenkapitel eingeräumt. Wer spricht als Chirurg schon gerne über seine Komplikationen? Für die betroffenen Patienten stehen diese aber je nach Schweregrad oft ganz im Vordergrund. Sie belasten die Genesung und hinterlassen mitunter lebenslange negative Folgen.

\section{( $)$ Zahl und Schwere der Kompli- kationen in der Rekonstruktions- chirurgie reduzieren}

Wer genauer hinsieht erkennt, dass die Beschäftigung mit den Komplikationen im Zeitalter der hochentwickelten Chirurgie ein Schlüssel zum Erfolg ist. Wichtiger als wieder eine neue Endoprothese zu testen wird es, Zahl und Schwere der Komplikationen weiter zu reduzieren. Dazu müssen wir diese systematisch erfassen, zählen und analysieren. Dann können wir vergleichen, informieren und verbessern. Damit können wir auch berechtigten Forderungen von Patienten und Po- litik nach Qualitätssicherung und -verbesserung gerecht werden.

Im Rahmen dieses Heftes mussten Schwerpunkte gesetzt werden. Diese orientieren sich an der Häufigkeit (Hüftoperationen, Thrombose), dem möglichen Schweregrad (Wirbelsäulenoperationen) oder der Bedeutung (Infektion, Transfusionsreaktion) bestimmter Komplikationen.

Therapieerfolge und Komplikationen werden auch durch Begleiterkrankungen wie den Diabetes mellitus, Thrombophilie oder neurologische Erkrankungen spezifisch beeinflusst. Folglich müssen wir uns mit diesen Erkrankungen in Klinik und Praxis intensiv beschäftigen.

Ich danke den Herausgebern und dem Verlag, dass sie diesem Komplex ein Themenheft gewidmet haben und hoffe auf eine anregende Wirkung im klinischen Alltag und in der wissenschaftlichen Diskussion. Es gibt eine Vielfalt von Themen in diesem Zusammenhang, die auf eine weitere Betrachtung, Aus- und Bewertung warten.<smiles>CC1(C)CCCC1</smiles>

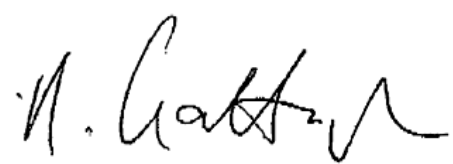

B.-D. Katthagen

\section{Korrespondenzadresse \\ Prof. Dr. B.-D. Katthagen}

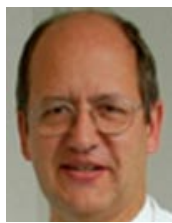

Orthopädische Klinik

Klinikum Dortmund

Beurhausstraße 40,

44137 Dortmund

b.d.katthagen@klinikumdo.de 